\title{
Editorial
}

\section{Teaching Cardiology}

\author{
Kempson MADDox, M.D.*
}

$\mathrm{T}$ HE vast majority of patients suffering from cardiovascular disease are not admitted to hospitals, except terminally, but are cared for by the general practitioner, and altogether they constitute a very large segment of his practice. It is his duty to detect cardiovascular disease at an early stage, to offer advice as to its prevention, deceleration, treatment and rehabilitation. It is the duty of teachers of students and post graduates to ensure that he is prepared to do this in an intelligent, dedicated and efficient manner. By a false diagnosis, a practitioner can create the dark and everlasting shadow of fear over his patient and family, resulting in years of unwarranted cardiac invalidism. By intelligent and informed observation, he may be able to dispel such a false opinion, to his own great satisfaction and to the eternal gratitude of the patient and his family.

One of the most fascinating attractions of cardiology is its close relationship to other areas of medicine, and this overlap increases year by year. The training of a modern specialist in cardiovascular diseases included a study of radiology, electronics, metabolism, biochemistry, epidemiology, haemodynamics, genetics, respiratory function, cytology and pharmacology. So extensive indeed is this field that it is beyond the ability of one individual to encompass it, and a whole team of workers must unite to represent the old fashioned cardiologist and distil for the general practitioner certain conclusions of practical value to him.

The medical student, quite rightly, learns something of each of these areas from teachers of physics, physiology, biochemistry, public health and so forth, but there is a tendency all over the world to demand from him considerable technical and mathematical knowledge, to the detriment of his interest and to the exclusion of main principles and points of cohesion between these various disciplines.

The student is impatient to enter the hospital ward and often regards this basic knowledge as inevitable "rough ground" to be treaversed as soon as possible and as superficially as is necessary to pass the requisite examina-

\footnotetext{
* 141 Macquarie Street, Sydney, Australia.
} 
tions and then forgotten in the excitement of his first contact with patients. In some medical schools of the British Commonwealth, intelligent students are invited to spend a further year in the basic Science Departments, with the attraction of a Science Degree, and a few, with an interest already wakened in Research, accept this opportunity. The National Heart Foundation of Australia assists them financially during this year. The Foundation also pays other students to spend some of their vacation time in laboratories involved in cardiovascular problems. In this way future research workers may realize early attraction of an academic or investigational career.

The remainder enter the clinical section of their career, and begin to learn the elements and routine of clinical diagnosis. It is here that a tremendous responsibility devolves upon the clinical teachers, who must strive for an intelligent and realistic transition from the normal to the abnormal. They must see that the student retains his respect and knowledge of applied physiology, anatomy and biochemistry, at the same time exposing to him the changes induced by disease, in its natural history, and by a relevation of the mechanisms by which disease interferes with normal physiology and biochemistry. Naturally, he must be taught his tools of trade, the correct techniques in the use of his hands, eyes and ears and even his nose, his stethoscope, sphygnomanometer, ophthalmoscope etc., how to appreciate the background of disease, the social and psychological factors involved, and how to take a proper systematic and consecutive case history. Among the greatest contributions of the British and French Schools of cardiology are those of semiology and of accurate and painstaking bedside diagnosis. Today in some quarters these things are belittled by the new generation of physician in the frantic search for precise quantitation. Certainly modern laboratory techniques of haemodynamics, and many forms of vivo quantitation have done something to correct bedside findings, but such has also been confirmed, and the two sources of information are complementary. A general practitioner cannot carry about with him a cardiac catheter, a densitometer or an X-ray fluoroscope, but he can always have his eyes, ears and hands, and he should be trained to extract the utmost from their use, both in history taking and in clinical examination.

Specialists inevitably tend to teach students knowledge which is too specialized, and methods of examination which cannot be applied in the home. They must learn to think in terms of the maximum information an isolated practitioner can elicit, and deliberately refrain from teaching in any detail anything else. They must teach principles such as mechanisms of disturbed physiology, the natural history and the classical clinical syndromes of heart disease, and their treatment, the recognition of occult diseases of the heart and blood vessels, the social and psychological factors involved and their influence 
on the natural history of heart discase, the use and limitations of classical drugs and the principles of rehabilitation. The place of cardiovascular surgery and its limitations and dangers must be explained. The students should be urged to read certain classical descriptions of heart disease from the original articles, but also taught how to make best use of a modern medical library. Medical journal reading should be encouraged, and a determined effort made to develop a critical faculty even in an undergraduate. Should a patient be allotted to a particular student fail to recover, the student must attend the autopsy, bring all the details of this examination to his teacher and his class, and attempt to correlate the clinical signs and history with the post mortem findings. Similar comparisons of clinical with X-ray or laboratory results should be insisted upon. All this is intended to emphasize the extraordinary distance toward diagnosis and prognosis with meticulous and systematic clinical examination may take the careful examiner.

Such a discipline, insisted upon from the beginning, will of course, assist in the recognition of other non-cardiac pathology. The embryo cardiologist must learn to make a full and detailed examination of all the remaining bodily symptoms. In fact his first exercise should be a general survey of the patient's nutrition, colour, posture, gait, skin, and behaviour from afar, long before reaching his stethoscope. To fail to recognize the co-existence of anaemia, thyroid disorder, chronic alcoholism, vitamin deficiency etc., may lose him golden opportunities for successful therapy.

Good teaching depends more on the teacher than the student. Enthusiams, patience, and forethought make the successful medical instructor.

If the student has been taught to have confidence in his own senses, to think " how" and "why " a certain symptom or sign has arisen, to be curious and to ask questions of others or from books, to be able to select patients requiring more expert help, then he is well on the road to becoming a dependable cardiologist in general practice. 